\title{
ON THE INDECOMPOSABLE ELEMENTS OF THE BAR CONSTRUCTION
}

\author{
RICHARD M. HAIN ${ }^{1}$
}

\begin{abstract}
An explicit formula for a canonical splitting $s: Q \mathscr{B}\left(\mathscr{E}^{\circ}\right) \rightarrow \mathscr{B}\left(\mathscr{E}^{\circ}\right)$ of the projection $\mathscr{B}\left(\mathscr{E}^{\circ}\right) \rightarrow Q \mathscr{B}\left(\mathscr{E}^{\circ}\right)$ of the bar construction on a commutative d.g. algebra $\mathscr{E}^{\circ}$ onto its indecomposables is given. We prove that $s$ induces a d.g. algebra isomorphism $\Lambda\left(Q \mathscr{B}\left(\mathscr{E}^{\circ}\right)\right) \rightarrow \mathscr{B}\left(\mathscr{E}^{\circ}\right)$ and that $H\left(Q \mathscr{B}\left(\mathscr{E}^{\circ}\right)\right)$ is isomorphic with $Q H\left(\mathscr{B}\left(\mathscr{E}^{\circ}\right)\right)$.
\end{abstract}

If $\mathscr{E}^{\circ}$ is an augmented commutative d.g. algebra over a field $k$ of characteristic zero, then the bar construction $\mathscr{B}\left(\mathscr{E}^{\circ}\right)$ on $\mathscr{E}^{\bullet}$ is a commutative d.g. Hopf algebra. Denote the augmentation ideal of $\mathscr{B}\left(\mathscr{E}^{\circ}\right)$ by $I \mathscr{B}\left(\mathscr{E}^{\circ}\right)$ (or $I$ ). The indecomposable elements of $\mathscr{B}\left(\mathscr{E}^{\circ}\right)$ are defined to be $Q \mathscr{B}\left(\mathscr{E}^{\circ}\right)=I / I^{2}$. Since $\mathscr{B}\left(\mathscr{E}^{\circ}\right)=k \oplus I \mathscr{B}\left(\mathscr{E}^{\circ}\right)$, there is a natural projection $\mathscr{B}\left(\mathscr{E}^{\circ}\right) \rightarrow Q \mathscr{B}\left(\mathscr{E}^{\circ}\right)$. Our main result is the following.

THEOREM. If $\mathscr{E}^{\bullet}$ is a commutative d.g. algebra over the field $k$ of characteristic zero, then there is a natural splitting $s: Q \mathscr{B}\left(\mathscr{E}^{\circ}\right) \rightarrow I \mathscr{B}\left(\mathscr{E}^{\circ}\right)$ of the natural projection $\pi: I \mathscr{B}\left(\mathscr{E}^{\circ}\right) \rightarrow Q \mathscr{B}\left(\mathscr{E}^{\circ}\right)$. The splitting s commutes with the differentials. Moreover, the map $\Lambda\left(Q \mathscr{B}\left(\mathscr{E}^{\circ}\right)\right) \rightarrow \mathscr{B}\left(\mathscr{E}^{\circ}\right)$ induced by $s$, from the free commutative d.g. algebra generated by $Q \mathscr{B}\left(\mathscr{E}^{\circ}\right)$ into $\mathscr{B}\left(\mathscr{E}^{\circ}\right)$, is a d.g. algebra isomorphism.

In fact, the idempotent $\gamma=s \circ \pi$ is given by the formula

$$
\gamma\left[a_{1}|\cdots| a_{n}\right]=\sum_{m=1}^{n} \sum_{r_{1}+\cdots+r_{m}=n} \sum_{\sigma \in \operatorname{sh}\left(r_{1}, \ldots, r_{m}\right)}(-1)^{m-1} \frac{\varepsilon(\sigma)}{m}\left[a_{\sigma(1)}|\cdots| a_{\sigma}(n)\right],
$$

where $\operatorname{sh}\left(r_{1}, \ldots, r_{m}\right)$ denotes the shuffles of $\{1, \ldots, m\}$ of type $\left(r_{1}, \ldots, r_{m}\right)$ and where $\varepsilon: \Sigma_{m} \rightarrow\{-1,1\}$ is the representation of the symmetric group obtained by assigning weight $-1+\operatorname{deg} a_{j}$ to $a_{j}$.

COROllaRY. The natural map $Q H^{\cdot}\left(\mathscr{B}\left(\mathscr{E}^{\circ}\right)\right) \rightarrow H^{\cdot}\left(Q \mathscr{B}\left(\mathscr{E}^{\circ}\right)\right)$ is an isomorphism.

One version of the Poincaré-Birkhoff-Witt (P.B.W.) theorem (cf. [6, appendix B]) states that if $A$ is a commutative d.g. Hopf algebra, then there is a natural d.g. coalgebra isomorphism $S(P A) \approx A$ between $A$ and the symmetric coalgebra on the primitives $P A$ of $A$. Thus, the assertion that there is a d.g. algebra isomorphism

Received by the editors March 20, 1985.

1980 Mathematics Subject Classification (1985 Revision). Primary 55P62.

Key words and phrases. Bar construction.

${ }^{1}$ Supported in part by grants MCS-8201642 and DMS-8401175 from the National Science Foundation. 
$\Lambda\left(Q_{B}\left(\mathscr{E}^{\circ}\right)\right) \approx \mathscr{B}\left(\mathscr{E}^{\circ}\right)$ is, in some sense, a dual P.B.W. theorem (cf. [4]). In fact, if $\mathscr{E}^{\bullet}$ is 1-connected and of finite type, then $\mathscr{B}\left(\mathscr{E}^{\circ}\right)$ is also of finite type and this assertion follows from the P.B.W. theorem by taking $A=\operatorname{Hom}\left(\mathscr{B}\left(\mathscr{E}^{\circ}\right), k\right)$. When $\mathscr{E}^{\bullet}$ is not connected or not of finite type, $\mathscr{B}\left(\mathscr{E}^{\circ}\right)$ is no longer of finite type and such a dualization argument cannot be applied directly because the dual of $\mathscr{B}\left(\mathscr{E}^{\circ}\right)$ is not a Hopf algebra.

The interesting part of the theorem is the formula for the splitting. This has been used in [3] where it is used to prove the technical, but useful result that if $\mathscr{E}^{\circ}$ is a mixed Hodge complex (M.H.C.), then $Q \mathscr{B}\left(\mathscr{E}^{\circ}\right)$ is also a M.H.C. It was decided to publish the result of this paper separately since it may be of wider interest.

The proof of the theorem is inspired by K.-T. Chen's method of power series connections and the relationship between iterated integrals and the bar construction on the one hand and iterated integrals and the Lie theory of nilpotent groups on the other (cf. [1]).

1. The bar construction. Suppose that $\mathscr{A}^{\circ}$ is a (not necessarily commutative) differential graded algebra (d.g.a.) with augmentation ideal $I_{\mathscr{A}}{ }^{\circ}$. It is convenient to define

$$
J a=(-1)^{\operatorname{deg} a} a
$$

for all $a \in \mathscr{A}^{*}$. The bar construction on $\mathscr{A}^{\cdot}$ is the double complex $\oplus B^{s, t}$, where

$$
B^{-s, t}=\left(\otimes^{s} I \mathscr{A}^{\cdot}\right)^{t} \text {. }
$$

A typical element of $B^{-s, t}$ will be denoted $\left[a_{1}\left|a_{2}\right| \cdots \mid a_{s}\right]$, where $a_{j} \in I \mathscr{A}^{\circ}$. The external differential $d_{E}: B^{-s, t} \rightarrow B^{-s, t+1}$ is defined by

$$
d_{E}:\left[a_{1}|\cdots| a_{s}\right] \mapsto \sum_{i=1}^{s}(-1)^{i}\left[J a_{1}|\cdots| J a_{i-1}\left|d a_{i}\right| \cdots \mid a_{s}\right] .
$$

The internal differential $d_{I}: B^{-s, t} \rightarrow B^{-s+1, t}$ is defined by

$$
d_{l}:\left[a_{1}|\cdots| a_{s}\right] \mapsto \sum_{i=1}^{s-1}(-1)^{i+1}\left[J a_{1}|\cdots| J a_{i-1}\left|J a_{i} \wedge a_{i+1}\right| \cdots \mid a_{s}\right] \text {. }
$$

One can check that $d_{I} d_{E}+d_{E} d_{I}=0$. When we refer to the bar construction, we will mean the associated total complex $\mathscr{B}\left(\mathscr{A}^{\circ}\right)=\left(\oplus B^{s, t}, d_{I}+d_{E}\right)$. With the diagonal

$$
\begin{gathered}
\Delta: \mathscr{B}\left(\mathscr{A}^{*}\right) \rightarrow \mathscr{B}\left(\mathscr{A}^{\circ}\right) \otimes \mathscr{B}\left(\mathscr{A}^{\circ}\right), \\
{\left[a_{1}|\cdots| a_{s}\right] \mapsto \sum_{i=0}^{s}\left[a_{1}|\cdots| a_{i}\right] \otimes\left[a_{i+1}|\cdots| a_{s}\right],}
\end{gathered}
$$

$\mathscr{B}\left(\mathscr{A}^{\circ}\right)$ becomes a d.g. coalgebra. If $\mathscr{A}^{\circ}$ is also commutative, then equipped with the shuffle product

$$
\begin{gathered}
\wedge: \mathscr{B}\left(\mathscr{A}^{\circ}\right) \otimes \mathscr{B}\left(\mathscr{A}^{\circ}\right) \rightarrow \mathscr{B}\left(\mathscr{A}^{\circ}\right), \\
{\left[a_{1}|\cdots| a_{r}\right] \otimes\left[a_{r+1}|\cdots| a_{r+s}\right] \mapsto \sum_{\sigma} \varepsilon(\sigma)\left[a_{\sigma(1)}|\cdots| a_{\sigma(r+s)}\right],}
\end{gathered}
$$

where $\sigma$ ranges over all shuffles of type $(r, s)$ and where $\varepsilon(\sigma)$ is $\pm 1, \mathscr{B}\left(\mathscr{A}^{*}\right)$ becomes a d.g. Hopf algebra. 
2. Proof of the theorem. First consider the case where the underlying algebra of $\mathscr{E}^{\bullet}$ is the free graded commutative algebra $\Lambda\left(a_{1}, \ldots, a_{n}\right)$, where $\operatorname{deg} a_{j} \geqslant 2$ for each $j$. The bar construction $\mathscr{B}\left(\mathscr{E}^{\circ}\right)$ is of finite type and so $A=\operatorname{Hom}\left(\mathscr{B}\left(\mathscr{E}^{\circ}\right), k\right)$ is a cocommutative d.g. Hopf algebra. From the P.B.W. theorem and the Milnor-Moore theorem [5], we know that $A$ is isomorphic, as a d.g. coalgebra, with the free symmetric coalgebra $S(P A)$ on the set of primitives $P A$ of $A$. The dual of the natural projection $p: \overline{S(P A)} \rightarrow P A$ is the map $s: Q \mathscr{B}\left(\mathscr{E}^{\circ}\right) \rightarrow I \mathscr{B}\left(\mathscr{E}^{\circ}\right)$ that we seek, and the d.g. algebra map $\Lambda s: \Lambda\left(Q \mathscr{B}\left(\mathscr{E}^{\circ}\right)\right) \rightarrow \mathscr{B}\left(\mathscr{E}^{\circ}\right)$ induced by $s$ is dual to the d.g. coalgebra isomorphism $A \rightarrow S(P A)$. Consequently, $\Lambda s$ is an isomorphism of d.g. algebras.

The next step is to derive the formula for the associated idempotent $\gamma: I \mathscr{B}\left(\mathscr{E}^{\circ}\right) \rightarrow$ $I \mathscr{B}\left(\mathscr{E}^{\circ}\right)$ in this case. For the time being, we will forget about differentials. Choose a graded basis $b_{1}, b_{2}, \ldots$ of $I \mathscr{E}^{\bullet}$ with $b_{j}=a_{j}$ when $1 \leqslant j \leqslant n$. Denote the dual basis of $\operatorname{Hom}\left(I \mathscr{E}^{\circ}, k\right)$ by $X_{1}, X_{2}, \ldots$ Desuspend (i.e., reduce the degree of) each $X_{j}$ by 1 . Denote the free graded associative ring generated by $X_{1}, X_{2}, \ldots$ by $k\left\langle X_{1}, X_{2}, \ldots\right\rangle$ and the ring of formal power series in the indeterminates $X_{1}, X_{2}, \ldots$ with coefficients in $\mathscr{B}\left(\mathscr{E}^{\circ}\right)$ by $\mathscr{B}\left(\mathscr{E}^{\circ}\right)\left\langle\left\langle X_{1}, X_{2}, \ldots\right\rangle\right\rangle$. By defining each $X_{j}$ to be primitive, these algebras become (complete) Hopf algebras. ${ }^{2}$

The key to establishing the formula for $\gamma$ is to view a certain element of $\mathscr{B}\left(\mathscr{E}^{\circ}\right)\left\langle\left\langle X_{1}, X_{2}, \ldots\right\rangle\right\rangle$ as a Hopf algebra isomorphism between $k\left\langle X_{1}, X_{2}, \ldots\right\rangle$ and $A$. Viewing the isomorphism in this way allows one to take its logarithm, which turns out to be the correct thing to do.

Consider the element

$$
T=1+\sum\left[b_{j}\right] X_{j}+\sum\left[b_{i} \mid b_{j}\right] X_{i} X_{j}+\sum\left[b_{i}\left|b_{j}\right| b_{k}\right] X_{i} X_{j} X_{k}+\cdots
$$

of $\mathscr{B}\left(\mathscr{E}^{\circ}\right)\left\langle\left\langle X_{1}, X_{2}, \ldots\right\rangle\right\rangle$. An element $\varphi$ of $A=\operatorname{Hom}\left(\mathscr{B}\left(\mathscr{E}^{\circ}\right), k\right)$ can be evaluated on $T$ to give an element $\langle T, \varphi\rangle$ of $k\left\langle X_{1}, X_{2}, \ldots\right\rangle$. That is, $T$ defines a linear map

$$
\theta: A \rightarrow k\left\langle X_{1}, X_{2}, \ldots\right\rangle, \quad \varphi \mapsto\langle T, \varphi\rangle .
$$

In fact, $\theta$ is a graded Hopf algebra isomorphism (cf. (6.17), [2]).

By the usual computation $T=\exp (\log T)$ in $\mathscr{B}\left(\mathscr{E}^{\circ}\right)\left\langle\left\langle X_{1}, X_{2}, \ldots\right\rangle\right\rangle$. It follows from formula (b) [2, p. 58] that $T$ is a group-like element of the complete Hopf algebra $\mathscr{B}\left(\mathscr{E}^{\circ}\right)\left\langle\left\langle\mathrm{X}_{1}, X_{2}, \ldots\right\rangle\right\rangle$. That is, $\Delta T=T \hat{\otimes} T$. It follows that $\log T$ is primitive. That is,

$$
\Delta \log T=\log T \hat{\otimes} 1+1 \hat{\otimes} \log T .
$$

Consequently, the linear map $A \rightarrow k\left\langle X_{1}, X_{2}, \ldots\right\rangle: \varphi \mapsto\langle\log T, \varphi\rangle$ lands in the set of primitive elements $P$ of $k\left\langle X_{1}, X_{2}, \ldots\right\rangle$.

The isomorphism $k\left\langle X_{1}, X_{2}, \ldots\right\rangle \rightarrow S(P)$ induces a direct sum decomposition

$$
k\left\langle X_{1}, X_{2}, \ldots\right\rangle=k \oplus P \oplus S^{2}(P) \oplus S^{3}(P) \oplus \cdots,
$$

where $S^{m}(P)$ denotes the symmetric tensors of rank $m$ on $P$. It is not hard to check, because $\mathscr{B}\left(\mathscr{E}^{\bullet}\right)$ is commutative, that the map $A \rightarrow k\left\langle X_{1}, X_{2} \ldots\right\rangle: \varphi \mapsto\left\langle(\log T)^{m}, \varphi\right\rangle$

\footnotetext{
${ }^{2}$ Note that here we are regarding $\mathscr{B}\left(\mathscr{E}^{\circ}\right)$ as an ungraded ring. In particular, the coefficients of a powerseries commute with the $X_{j}$ when powerseries are being multiplied.
} 
lands in $S^{m}(P)$. Since $T=\exp (\log T)$, it follows that the composite $I A \rightarrow$ $I k\left\langle X_{1}, X_{2}, \ldots\right\rangle \stackrel{p}{\rightarrow} P$ takes $\varphi$ to $\langle\log T, \varphi\rangle$. Since the diagram

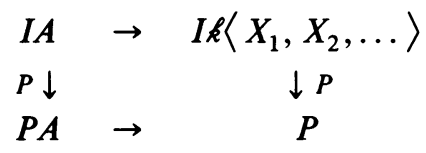

commutes, and since $\gamma$ is dual to $p: I A \rightarrow P A$, it follows that

$$
\log T=\sum \gamma\left[b_{j}\right] X_{j}+\sum \gamma\left[b_{i} \mid b_{j}\right] X_{i} X_{j}+\sum \gamma\left[b_{i}\left|b_{j}\right| b_{k}\right] X_{i} X_{j} X_{k}+\cdots
$$

But

$$
\begin{aligned}
& \log T=\sum_{m=1}^{\infty} \frac{(-1)^{m-1}}{m}(T-1)^{m} \\
& \vdots \\
&=\sum_{n=1}^{\infty} \sum_{\left(j_{1}, \ldots, j_{n}\right)} \sum_{m=1}^{n} \sum_{r_{1}+\cdots+r_{m}=n} \sum_{\sigma \in \operatorname{sh}\left(r_{1}, \ldots, r_{m}\right)}(-1)^{m-1} \frac{\varepsilon(\sigma)}{m} \\
& {\left[b_{j_{\sigma(1)}}|\cdots| b_{j_{(n)}}\right] X_{j_{1}} X_{j_{2}} \cdots X_{j_{n}} . }
\end{aligned}
$$

Comparing the coefficients of $X_{1} X_{2} \cdots X_{n}$ we see that

(*)

$$
\begin{aligned}
& \gamma\left[a_{1}|\cdots| a_{n}\right]= \sum_{m=1}^{n} \sum_{r_{1}+\cdots+r_{m}=n} \frac{(-1)^{m-1}}{m}\left[a_{1}|\cdots| a_{r_{1}}\right] \wedge\left[a_{r_{1}+1}|v \cdots| a_{r_{2}}\right] \\
& \cdots \wedge\left[a_{n+1-r_{n}}|\cdots| a_{n}\right] \\
&=\sum_{m=1}^{n} \sum_{r_{1}+\cdots+r_{m}=n} \sum_{\sigma \in \operatorname{sh}\left(r_{1}, \ldots, r_{m}\right)}(-1)^{m-1} \frac{\varepsilon(\sigma)}{m}\left[a_{\sigma(1)}|\cdots| a_{\sigma(n)}\right] .
\end{aligned}
$$

Now suppose that $\mathscr{E}^{\bullet}$ is an arbitrary commutative d.g. algebra. Define $\gamma$ : $I \mathscr{B}\left(\mathscr{E}^{\circ}\right) \rightarrow I \mathscr{B}\left(\mathscr{E}^{\circ}\right)$ using the formula above. It is immediate that $\gamma=\gamma^{2}$, that $\gamma$ commutes with the differential and that $\gamma$ is a graded map of degree 0 .

If $a_{1}, \ldots, a_{n} \in I \mathscr{E}^{\circ}$, then we can find a free graded commutative algebra $\mathscr{A}^{\circ}=$ $\Lambda\left(\bar{a}_{1}, \ldots, \bar{a}_{n}\right)$ where

(a) $\operatorname{deg} \bar{a}_{j} \geqslant 2, j=1, \ldots, n$,

(b) $\operatorname{deg} \bar{a}_{j} \equiv \operatorname{deg} \bar{a}_{j} \bmod 2, j=1, \ldots, n$.

The natural map $\mathscr{A}^{\bullet} \rightarrow \mathscr{E}^{\bullet}$ defined by taking $\bar{a}_{j}$ to $a_{j}$ is $\mathrm{Z} / 2$ graded and thus induces a $\mathbf{Z} / 2$ graded Hopf algebra map $\mathscr{B}\left(\mathscr{A}^{\circ}\right) \rightarrow \mathscr{B}\left(\mathscr{E}^{\circ}\right)$ that commutes with $\gamma$. The assertions of the theorem now follow. For example, we will prove that $\operatorname{ker} \gamma=I^{2} \mathscr{B}\left(\mathscr{E}^{\circ}\right)$.

First, if $u \in I^{2} \mathscr{B}\left(\mathscr{E}^{\circ}\right)$, then there exists a subalgebra $\mathscr{F}^{\cdot}$ of $\mathscr{E}^{\bullet}$ generated by $a_{1}, \ldots, a_{n}$ say, such that $u \in I^{2} \mathscr{B}\left(\mathscr{F}^{\circ}\right)$. Pick a free graded commutative algebra $\mathscr{A}^{\circ}=\Lambda\left(\bar{a}_{1}, \ldots, \bar{a}_{n}\right)$ as above and a map $\mathscr{A}^{*} \rightarrow \mathscr{F}^{\circ}$. Denote by $\rho: \mathscr{B}\left(\mathscr{A}^{\circ}\right) \rightarrow \mathscr{B}\left(\mathscr{E}^{\circ}\right)$ the induced map. There exists $\hat{u} \in I^{2} \mathscr{B}\left(\mathscr{A}^{\circ}\right)$ such that $\rho(\hat{u})=u$. Since $\gamma(\hat{u})=0$ and $\rho \circ \gamma=\gamma \circ \rho$, it follows that $\gamma(u)=0$. That is, $I^{2} \mathscr{B}\left(\mathscr{E}^{\circ}\right) \subseteq \operatorname{ker} \gamma$. On the other hand, it follows directly from (*) that $\operatorname{im}(\gamma-1) \subseteq I^{2} \mathscr{B}\left(\mathscr{E}^{\circ}\right)$. Since $\gamma$ is an idempotent, $\operatorname{ker} \gamma=\operatorname{im}(1-\gamma)$. Thus $\operatorname{ker} \gamma \subseteq I^{2} \mathscr{B}\left(\mathscr{E}^{\circ}\right)$. 
The assertion that the map $\Lambda s: \Lambda\left(Q \mathscr{B}\left(\mathscr{E}^{\circ}\right)\right) \rightarrow \mathscr{B}\left(\mathscr{E}^{\circ}\right)$ is a d.g. algebra isomorphism is proved similarly.

REMARKS. (a) The referee has pointed out that since only the differential of $B\left(\mathscr{A}^{\circ}\right)$ depends on the multiplication of $\mathscr{A}^{\circ}$, all assertions of the theorem, with the exception of the statement that $d s=d s$, follow from the corresponding assertions for algebras with trivial multiplication. Since these are direct limits of their finite dimensional subalgebras, we may assume that $\mathscr{A}$ is finite dimensional. Taking the bigraded dual of $B\left(\mathscr{A}^{\circ}\right)$, one sees that the existence of the splitting $s$ and the fact that $\Lambda s$ is an isomorphism are immediate consequences of the P.B.W. theorem.

(b) An immediate consequence of the proof of the theorem is that we now have an explicit formula for the Lie transport $T_{L}$ of a formal connection $(\omega, L)$ on a d.g.a. $\mathscr{E}^{\bullet}$ (cf. [2, (6.19)]). If $T=1+[\omega]+[\omega \mid \omega]+\cdots$ is the usual formal transport of $T$, then $T_{L}=\log T$.

\section{REFERENCES}

1. K.-T. Chen, Iterated path integrals, Bull. Amer. Math. Soc. 83 (1977), 831-879.

2. R. M. Hain, Iterated integrals and homotopy periods, Mem. Amer. Math. Soc. No. 291, 1984.

3. The de Rham homotopy theory of complex algebraic varieties, preprint, 1984.

4. W. Michaelis, The dual Poincaré-Birkhoff-Witt Theorem, Adv. in Math. (to appear).

5. J. Milnor and J. Moore, On the structure of Hopf algebras, Ann. of Math. (2) 81 (1965), 211-264.

6. D. Quillen, Rational homotopy theory, Ann. of Math. (2) 90 (1969), 205-295.

Department of Mathematics, University of Washington, Seattle, Washington 98195 